\title{
Physicians' attitudes regarding the disclosure of medical errors: A qualitative study
}

\author{
Vitor S. Mendonca, Maria Luisa S. Schmidt \\ Institute of Psychology, University of Sao Paulo, Brazil
}

\begin{abstract}
The experiences of physicians' errors could affect their professional practice. The aim of study was to explore physicians' experiences of medical errors and its consequences. This was a qualitative study in which ten Brazilian physicians were selected through purposive sampling. The data were collected via semistructured interviews and analyzed through principles derived of the phenomenological method. The interview topics were around how the error occurred in their practice, its process of disclosure and consequences of medical errors. Excessive workload and difficulty communicating among physicians are some of the factors that may increase the likelihood of medical error. Not looking for help after the error was a common attitude. This may be related to the difficulty for some physicians to acquire new practices after the error, even though physicians have shown that experiencing a medical error situation has a negative impact in their lives. To disclose training and institutional practices which contribute to the dissemination of conduct favorable to the improvement of medical practice.
\end{abstract}

\footnotetext{
Correspondence: Vitor S. Mendonca, University of Sao Paulo/Brazil Institute of Psychology, Av Professor Mello de Moraes, 1721, bloco A, Caixa postal 66.261 CEP 05508-900, Sao Paulo-SP, Brazil.

E-mail: vitor.pospsico@bol.com.br
}

Key words: Medical error, disclosure, attitudes, physician.

Acknowledgment: We would like to thank all the participants and the hospital for the knowledge and support that they contributed to this study.

Contributions: VSM, literature survey, writing the review, corrections; MLSS, editing the review, corrections.

Conflict of interest: The authors declare no conflict of interest.

Funding: This study was funded by Sao Paulo Research Foundation [2015/09289-9 and 2016/23681-1].

Received for publication: 28 February 2020.

Revision received: 20 May 2020.

Accepted for publication: 21 May 2020.

This work is licensed under a Creative Commons AttributionNonCommercial 4.0 International License (CC BY-NC 4.0).

${ }^{\circ}$ Copyright: the Author(s), 2020

Licensee PAGEPress, Italy

Health Psychology Research 2020; 8:8929

doi:10.4081/hpr.2020.8929

\section{Introduction}

In many countries, physicians and health care institutions need help to disclose medical errors and in Brazil this is the same. In Brazil, patient safety and quality service are not primordial aspects of the medical practice. For this reason, the consequences of medical errors to the parties involved lead to situations that may lower the quality of the medical services provided. Communication, transparency and disclosure of errors should be the main characteristics of quality health care. However, there has been little debate on error disclosure and its importance to physicians. Moreover, there are some other challenges to Brazilian Medicine, such as the error-free medical practice culture, the lack of resources for disclosure and the tendency to condemn physicians who made mistakes by considering them evil professionals (Giostri, 2002; Mendonca, Gallagher \& Oliveira, 2018; Mendonca \& Schmidt, 2019).

The debate on medical error has become more notorious due to the exponential increase in the number of victims in different countries. Furthermore, the advent of new medical technologies has increased the variety of the errors, which lead to discussions about the medical service provided to patients, since in Brazil and some other countries, medical training is known for the search for infallibility and for considering medical errors to be unacceptable (Mendonca, et al., 2018).

In the 1970 s, there was a significant increase in complaints against American physicians. In a ten-year period, the number of complains increased by $18 \%$. Monetary compensations followed a similar trend, going from 60 million USD to 5 billion USD in the same time frame (Hiatt, 1992). In the United Kingdom, 16\% of hospitalized patients had been affected by some kind of medical error until 2013 (Weerakkody et al., 2013). In France, one in ten patients have been affected by errors (Grange \& Papilon, 2008). There are no exact estimates of how many patients have been affected by errors in Brazil because there is no official service in charge of assessing this.

In Brazil, medical error is defined as "a failure to exercise the profession, resulting in a poor or adverse result by action or omission of the professional" (Giostri, 2002). In the Brazilian Code of Medical Ethics there is no definition in relation to the issue. However, Article 1 of Chapter III stresses that it is forbidden for the physician to "cause harm to the patient, by action or omission, characterized as malpractice, recklessness or negligence" (Federal Council of Medicine, 2009). In the United States, medical error is defined by the Institute of Medicine as a failure of a planned action to be completed as intended or as the use of a wrong plan to achieve an aim (Institute of Medicine (US) Committee on Quality of Health Care in America, Kohn, Corrigan, \& Donaldson, 2000).

Besides medical error being treated this way in some countries such as Brazil, the error disclosure process is not known or practiced by everyone. In the United States, the main health regulatory agencies as well as the medical profession itself endorse 
the practice of disclosure. The disclosure itself is the moment in which the medical professional acknowledges the occurrence of an error to the patient. Physicians should disclose detailed information, regardless of whether the patient asks, by means of an explicit statement that an error has occurred, including a description of the error, the reasons why it occurred, how recurrences will be prevented and, lastly, an apology (Gallagher, Waterman, Ebers, Fraser, \& Levinson, 2003; Gallagher, Studdert, \& Levinson, 2007; Troug, Browning, Johnson, \& Gallagher, 2011).

As health care practices do not prioritize quality service and patient safety, medical training favors aspects of infallibility, and medical errors are rarely disclosed, we consider it essential to study the experiences of Brazilian physicians who have had some kind of error happen in their professional practice.

\section{Methods}

\section{Study design}

This qualitative study utilized a descriptive, cross-sectional research design.

\section{Participants}

This is a study with ten Brazilian physicians who have experienced errors in their practices. Every participant is a physician in a private hospital located in the state of São Paulo/Brazil, which employs over six thousand physicians.

The chosen physicians have gone through the disclosure process between 2015 and 2017, and all of them are supposed to have upto-date medical licenses registered at the body that regulates the profession in Brazil. Professionals who have never experienced errors in their careers, which have disclosed them before 2015, because those are older experiences; or who have not gone through disclosure at the hospital being researched were not included in the study.

The physicians are between 36 and 61 years old. All of them are male. The average years of experience since graduation is 21.2, and their average weekly workload is 53 hours (Table 1).

\section{Data collection}

The study was completed between August 2016 and September
2018. Semi-structured interviews were made for this study. The questions concerned the following topics: concepts of error, how did the error occur in their practice, disclosure process and help, impact and consequences of medical errors; and changes in professional behavior after the error.

The meetings were recorded, including the full accounts provided. The transcription, analysis and interpretation were based on concepts and tenets derived from the existentialphenomenological theory.

\section{Data analysis}

Using the results obtained, the analysis was completed using the phenomenological method, which looks for the meaning and significance attributed by physicians to their routine experiences. The attempt to reach a universal explanation was abandoned in order to maintain the concrete experience that physicians face. The material was read on many occasions, during which written comments were made and significant sections were identified. Based on the analysis, a descriptive text was drafted, producing a dialog between the core identified themes and the references adopted by this study (Giorgi \& Sousa, 2010).

\section{Ethical consideration}

The ethics review board of the Brazilian human research committee approved the study. Physicians gave consent for their participation in this study. The participants were aware of the purpose of the study and they have the right to leave the study any time they will. They were assured that all their information would remain confidential. The physicians' anonymity was protected and safety of the material was maintained.

\section{Results}

\section{How physicians conceptualize and characterize medical errors}

The conceptualization and characterization of medical error by those who have already experienced it is a tool to confront or confirm what theory tells us about this topic. The physicians of the study experience a paradox to characterize the medical error in Brazilian culture, because medical training is rooted on an error-

Table 1. Characteristics of participants.

\begin{tabular}{|c|c|c|c|c|}
\hline Name & Age & Specialty or area of practice & Graduation year & Workload \\
\hline D1 & 39 & Cardiology & 2000 & 80 \\
\hline D2 & 47 & Internal Medicine & 1995 & 45 \\
\hline D3 & 38 & Internal Medicine & 2002 & 50 \\
\hline D4 & 47 & Internal Medicine & 1998 & 60 \\
\hline D5 & 36 & Anesthesiology & 2007 & 45 \\
\hline D6 & 49 & Orthopedics & 2000 & 30 \\
\hline D7 & 41 & Surgery & 1999 & 60 \\
\hline D8 & 52 & Urology & 1990 & 60 \\
\hline D9 & 47 & Surgery & 1996 & 50 \\
\hline D10 & 61 & Anesthesiology & 1981 & 50 \\
\hline
\end{tabular}


free discourse, and the physicians in this study are outside the scope of what is socially acceptable.

"I think it is something very difficult to define, because we are educated in such a way during medical training that we try to minimize mistakes. And making fewer mistakes means expanding the diagnostic hypotheses and looking for conducts that cover most of these, without ignoring the most important ones. That is, infallibility is expected of us, even though practice is something altogether different" (D1)

Despite the difficulty, the group was able to define their concept of error. D2 could provide clear examples of some common situations among Brazilian physicians that explain frequent practical measures undertaken by physicians.

"Medical error is any mistake according to the patient, be it in diagnosis, be it in the treatment, that is, prescribing the wrong dose of some medicine or mistaking the pharmacological name of a drug for its commercial one, or a delay due to excessive workload concerning a patient. Another mistake is to not re-evaluate a patient or not giving clear information to the whole multi professional team on the case. This may occur due to lack of time or excessive workload. It may also occur when I notice a mistake, but do not disclose it for fear of being reprimanded by a more experienced colleague" (D2)

Participant D2 mentioned some elements which make the disclosure process more difficult and could even be considered socially-imposed barriers in the Brazilian medical profession: respect and fear of medical hierarchies and excessive physician workload.

\section{What happened?}

Every medical error of the participants occurred in the surgical environment. In general, errors are related to cardiac arrests during surgical procedures, out-of-date treatment that lead to the death of a patient, an arterial injury that compromised the blood flow and led to the loss of a limb, not following protocol, which lead to organ transplants between patients with different blood types, and cardiac arrest due to being unaware of drug allergies. Participant D7 tells us part of his case:

"It happened with a patient who suffered a complication in an ICU-procedure, which lead her to lose a leg. That was not her initial clinical condition. It was a sad situation, the family was unhappy and the severity of the case was explained.

She was admitted under my care, but this error happened due to a complication in the ICU. An arterial pulse occurred. That pulse ended up injuring the artery and stopping blood flow to the foot. Between the moments the diagnosis was initially made and the error was noticed, she ended up losing a leg" (D7)

\section{The disclosure process and the types of help}

In general, participants liked how the institution conducted the medical error and the disclosure process. They believe the institution has done its part regarding the error and listened to all involved parties before acting.

"I had the opportunity of experiencing an adverse situation in which the hospital took every viewpoint into account, which was a mature attitude. They had many professionals of related-fields to listen to each of the points of this event, and that had an interesting disclosure, and they understood my conduct. The support team and I understood the case better" (D1)

However, some aspects were not satisfactory, according to some participants. The physicians were not given a final report on the case. Some believe the process was quite slow and some also doubt that the institution had been neutral, favoring, instead, the patient's family.

"In general, the disclosure experience was not good. The overall feeling was that the second victim had been left out. The feeling is that we were neglected. The institution has its own interests. The institution talked about caring about this, but they did not act upon it" (D3)

"The disclosure happened with the risk management nurse, who came to me for an interview, but it took her quite a while to do so. It was not immediately after the event, no. Even though the hospital had said this was the fastest and most exceptional case in the institution" (D5).

A recurring issue in the speeches has been the judgments and self-judgments of the team involved and its professionals. Even though the institution practices the process of disclosure, it is evident that not only those involved in the events are made aware of the disclosure, but also many other people in the institution.

"In the months after the event, many people judged me, I was labeled as someone who could have had a different conduct in face of the disruptive behavior of that team considering what I wanted to do but failed to do so. During those years, I was scared of walking around in the hospital, because I wanted to avoid bumping into the family, who was around the hospital too" (D1)

Seeking and asking for help has not been recurrent. Most physicians claimed that they did not ask for help. However, those who did ask for help talk about how difficult it is to face the feeling of professional failure all by themselves. Families, bosses and psychologists were the ones physicians turned to for help the most.

"I myself decided to seek help, due to constant insomnia. My head would not stop. Therapy was very important to me, as it helped me question my feelings. It helped me face life, because I cannot change what happened. For many days, I did not want to come to work. I am afraid that this may happen again. I needed to understand that it was not directly my fault.

I talked to my boss, who helped me a lot. Look, I think that if I had not had disclosure, I would be much worse. Today, I can rest my head on the pillow and sleep properly. I know that the family is still angry, because they wanted the whole team fired" (D9)

\section{Impacts and consequences of medical errors to life}

In relation to the impressions caused by medical error in the personal and professional lives of the workers, it was possible to notice an initially negative impact which eventually lead to an attitude of reflection on subsequent actions, as an attempt to avoid new errors and also to change practical behavior.

"The anesthetic procedures after the event were terrible. I was scared and insecure. I doubled my attention after the event. When I finish, my surgery room looks exactly as it did when I entered. Everything is very well organized. 
I ended up becoming a safety-maniac. I try to foresee or anticipate every possibility. In the room, I test all equipment, as if it were my first day. Every syringe has been identified, to avoid medication-changing mistakes" (D10)

After the error, the health care institutions as well as the medical professionals have the opportunity of evaluating their practices and of devising new strategies. An interesting topic in this section is the role of protocols and the relation that physicians have with them. Initially, they do not seem to consider these protocols to be particularly important, but after the mistake, the book follows the protocols.

"See from a distance. See from proximity. Read again. If in doubt, involve other professionals. I have a very competent team and everything goes through a review system. If I have a surgery tomorrow, my anesthetist will talk to the patient on the day before. I always picture what might happen and protect myself. And I always explain. Attention to detail. Checking and double-checking. I try and follow these procedures, and keep myself up to date" (D8)

"Objectively follow all processes. I do everything according to this: checklist, double-checking and time out. The institution to prevent new errors established these protocols. The second thing is to worry about the patient. I do not need to be competing for patients in the hospital. The hospital competes for me. My bond to the patient is a big addition to my training. I am very transparent. And this bond helps me relax so I can talk to the patient and discuss things together" (D9)

However, not all professionals consider it necessary to change their practices after a medical error in their career. Some of them, a minority, believe that they do not need to re-evaluate their practice.

"No change in my practice. Here in the ICU we cannot think much. I need to do what I believe to be right, have confidence in my conduct. I continue study and always try to do my best. I am quite confident in how I work. There is no room to cry and be sad. My affection for the institution is still the same. What happened was just a gap" (D4).

Participant D4 is an example of how difficult it is to some Medicine professionals in Brazil, and perhaps other countries, to change their behavior. This happens because of they believe they do not need to change anything in their behavior or that they do not need extra care to avoid new mistakes.

\section{Discussion}

Medical training demands enormous dedication and effort by the physician-to-be. Countries such as the United States, Canada and France demand many more training hours than others such as Chile, India or Portugal, which often leads to discussions about the quality of the training. Excessive demand for technical promptness, greater responsibility regarding patient-care and pressure to have an error-free practice are demands made by some cultures which force the physician to be an error-free professional (Grange, \& Papilon, 2008; Galam, 2012). Medical education should prioritize two aspects: offering students the scientific and technical knowledge essential for the profession, and facilitating the maturing process of an adult and balanced personality, who is capable of understanding the complex biopsychosocial structure of the patient (Bitencourt, Neves, Neves, Brasil, \& Santos, 2007). The American Medical Association has published the Accelerating Change in Medical Education, elucidating aspects, which should necessarily be contemplated by American medical-training institutions. In general, it highlights improvements in the quality of medical attention, patient safety, care-based learning, population health and health care system knowledge, among others (American Medical Association, 2018).

Some studies have alerted to the high degree of emotional suffering physicians experience, which leads to a prevalence of anxiety and depression disorders, alcohol and drug abuse, and incidence of suicide. Excessive workloads, and working in multiple locations, as well as excessive weekly working hours, as observed on Table 1, have a direct impact on the social costs of the profession. Indeed, it has already been reported that the main cause of retirement among Brazilian physicians is mental disorders (Laranjeira, \& Alves, 2014; Stewart, et al., 1995).

Some training institutions have started initiatives to reduce the chance of becoming sick and of professional dysfunction among students, medical residents, and physicians (Barden, Specht, MacCarter, Daly, \& Fahey, 2002; Dunn, Moutier, Green Hammond, Lerhmann, \& Roberts, 2008; Goldstein, Kim, Widmann, \& Hardy, 2004). This reflects the concern of many countries regarding the mental health of health care workers, and the consequent prioritization of welfare, of an adequate workplace and of subjective risk reduction for physicians. According to the data of this study, it is possible to notice that medical hierarchies are a type of subjective risk imposed on Brazilian physicians, because there is an internal culture, in other words, corporatism, among peers, that the eldest doctor, and therefore the more experienced one, should not be confronted or opposed. This makes it more difficult for younger and less experienced physicians to exchange experiences or to question something in a large hospital, and it can even explain why it is difficult for physicians to seek help after experience a mistake, for example.

Another aspect of this study, which opposes the findings, and tendencies of universities and health care institutions throughout the world is the lack of orientation and health care of the physicians involved in the error, the so-called second victims. It is evident here that the hospital was not concerned and that the physicians were also unaware of the care and attention the institution should have paid its professionals. All of this reinforces the commercial thinking that the health of the population is something profitable, and that the health of the professionals is an afterthought.

In countries such as Brazil, training has been subject to plenty of criticism due to the need for change. The social pressure of errors being unacceptable and the demand of an error-free practice, as well as the questionable quality of education and the uncontrolled increase in the number of Medicine universities in the country are some of the elements of this discussion. Seeking help among peers is often considered as a sign of weakness among physicians. You are being educated in Medicine to be strong and to save your patient. Fragility and weakness must be hidden. Many professionals were not adequately stimulated to develop autonomy and the capacity of analyzing, judging and evaluating. Thus, an unprepared physician is sent to the labor market, unable to deal with mistakes in his practice (Gomes \& Rego, 2011). This prevents a constructive approach to the mistake, because peers and patients demand perfection.

It is important to note that in many countries, the governments are involved with the increasing number of medical schools. In France, the government established an entry-limit for universities 
in the 1980s. In Brazil, the government has authorized 218 new courses in the past 20 years, totaling 341 Medicine courses in 2019, a higher amount than in the United States or China. This has stimulated a debate. Often, this increase in the number of physicians who graduate from medical schools has not been sustained by the quality of teaching or quality facilities (Scheffer, 2013; Filho, \& Cassenote, 2018; Mendonca \& Schmidt, 2019).

To discuss and deal with medical error is complex and difficult. In some countries, this discussion is more advanced and disclosure is better structured. In many other countries, such as Brazil, this practice is still little known or used, which reinforces the innovative character of the initiative of the hospital in this study in developing a disclosure process; truly a pioneer movement in the country. Nevertheless, the disclosure process in many countries needs to be better discussed and studied in order to think of implementation possibilities (Mendonca, et al., 2018).

Medical error and disclosure processes should encourage an attitude of reflection and promote behavior change, both at a personal and at an institutional level. This should make health institutions rethink new practices and strategies. If disclosure is not yet used, trying and implement it might be a good idea. When disclosure is already used, it is necessary to continuously evaluate the applicability of the objectives because, as expressed by the study participants, there are still some questions regarding the process of disclosure implemented in the hospital under this study.

Of course, this is no easy task. Nevertheless, challenges offer the opportunity of thinking about how to overcome them, leading to initiatives to improve communication between physicians and patients by creating programs and training plans that include disclosure. Some of the challenges regarding disclosure are: the conflict between the theory of patient safety and the expectations of the patient, to put in practice good policies, the conflict of confidentiality, and the steps following disclosure. All of these challenges have been identified in countries such as the United States, England, Canada, New Zealand, and Australia, which have vast experience in error disclosure. This provides motivation to countries, which do not use the process or are still in the early stages of its implementation (Wu et al., 2017).

The disclosure process presented by the study participants is already an important tool for the hospital. Nonetheless, it needs to be improved. Physicians cannot question the neutrality of the institution nor have their own health care neglected. Institutions that promote disclosure need to make sure that the purpose of the process is not lost or mistaken with the possibility of avoiding a medical error leading to a lawsuit. The objectives of disclosure must be well established in the institution and be strictly followed, because judgments and bullying from other professionals to the medical team that implemented disclosure should not be allowed.

We need to understand that disclosure gives heath care institutions the possibility of considering errors as an opportunity to improve the system and, therefore, avoid similar occurrences in the future. Moreover, it improves patient security and significantly reduces the costs of medical assistance, it reduces patient suffering, alleviates physician suffering, fosters peer support, and reduces patient anger and frustration regarding the procedure or the lack of professional empathy (Troug, Browning, Johnson, \& Gallagher, 2011). All of these are incentives for countries such as Brazil, which need to widen the practice of disclosure in all medical instances.

Another finding was the difficulty in changing practices after the error. This does not happen in countries such as the United States, because the disclosure process makes physicians evaluate existing practices and devise new strategies to prevent the error from repeating. Thus, it guarantees learning and improvement for patients, families and physicians (Troug, Browning, Johnson, \& Gallagher, 2011).

One limitation of this study was the limited number of participants, mostly due to how difficult it is to have physicians who experienced errors accepts taking part in the study.

To conclude, medical training agents need to reconsider the process of Medical education. Physicians need to understand that, although medical error can be difficult, the act of disclosing instead of hiding the mistakes is ethically and legally appropriate in order to avoid further damage to the patient as well as to prevent further mistakes which the act of hiding could cause. Devising institutional practices and processes of disclosure and fostering actions that promote evolving patterns and behavior for the safety of patients in the country.

We recommend that disclosure be part of medical school curricula and medical residency programs. Promoting a culture of honesty and transparency can help guarantee the commitment of physicians to quality Medicine in countries where disclosure is not yet used. Where the process of disclosure is already being developed, this reinforces the opportunity of preventing future mistakes and increasing the quality of service. It is also recommended that health care institutions devise written policies and procedures describing how to reduce and disclose medical errors. We recommend that future studies research and compare information from more countries with different aspects, such as institutional disclosure or the lack thereof.

\section{References}

American Medical Association. (2018). Accelerating Change in Medical Education. [Accessed 12th December 2018] https:/www.ama-assn.org/education/accelerating-change-medicaleducation

Barden, C.B., Specht, M.C., MacCarter, M.D., Daly, J.M., \& Fahey, T.J. (2002). Effects of limited work hours on surgical training. Journal of the American College of Surgeons, 195(4), 531538 .

Bitencourt, A.G.V., Neves, N.M.B.C., Neves, F.B.C.S., Brasil, I.S.P.S., \& Santos, L.S.C. (2007). Análise do erro médico em processos ético-profissionais: implicações na educação médica. Revista Brasileira de Educação Médica, 31, 223-228.

Dunn, L.B., Moutier, C., Green Hammond, K.A., Lehrmann, J., \& Roberts, L.W. (2008). Personal Health Care of Residents: Preferences for Care Outside of the Training Institution. Academic Psychiatry, 32(1), 20-30.

Federal Council of Medicine. (2009). CFM Resolution no. 1931. September 17, 2009. Brasilia 4 p.

Filho, B.L., \& Cassenote, A.J.F. (2018). O Exame do CREMESP. Sao Paulo: Conselho Regional de Medicina do Estado de São Paulo.

Galam, E. (2012). L'Erreur médicale, le burnout et le soignant. Paris: Springer.

Gallagher, T.H., Studdert, D., \& Levinson, W. (2007). Disclosing harmful medical errors to patients. New England Journal of Medicine, 26, 2713-2719.

Gallagher, T.H., Waterman, A.D., Ebers, A.G., Fraser, V.J., \& Levinson, W. (2003). Patients' and physicians' attitudes regarding the disclosure of medical errors. JAMA, 289, 1001-1007.

Giorgi, A., \& Sousa, D. (2010). Método fenomenológico de investigação em Psicologia. Lisbon: Fim do seculo.

Giostri, H.T. (2002). Erro médico à luz da jurisprudência 
comentada. Curitiba: Juruá pp. 136.

Goldstein, M.J., Kim, E., Widmann, W.D., \& Hardy, M.A. (2004). A 360 degrees evaluation of a night- oat system for general surgery: a response to mandated work-hours reduction. Current Surgery, 61, 445-451.

Gomes, A.P., \& Rego, S. (2011). Transformação da educação médica: é possível formar um novo médico a partir de mudanças no método de ensino-aprendizagem? Revista Brasileira de Educação Médica, 35, 557-566.

Grange, P., \& Papilon, F. (2008). Erreurs médicales. Paris: Nil. Hiatt, H. (1992). Medical malpractice. Bulletin of the New York Academy of Medicine, 68(2), 254-264.

Institute of Medicine (US) Committee on Quality of Health Care in America, Kohn, L.T., Corrigan, J.M., \& Donaldson, M.S. (Eds.), (2000). To err is human: building a safer health system. Washington: National Academy Press.

Laranjeira, R., \& Alves, H. (2014). Médicos dependentes e com problemas psicológicos. Journal Cremesp 198.

Mendonca, V., \& Schmidt, M. (2019). Disclosure: possibility to discuss medical error. International Archives of Internal Medicine, $3,1-5$.

Mendonca, V., Gallagher, T., \& Oliveira, R. (2018). The
Function of Disclosing Medical Errors: New Cultural Challenges for Physicians. HEC Forum, 30.

Scheffer, M. (2013). Demografia médica no Brasil. São Paulo: Conselho Federal de Medicina e Conselho Regional de Medicina do Estado de São Paulo.

Stewart, S.M., Betson, C., Marshall, I., Wong, C.M., Lee, P.W., \& Lam, T.H. (2005). Stress and vulnerability in medical students. Medical Education, 29(2), 119-127.

Truog, R.D., Browning, D.M., Johnson, J.A., \& Gallagher, T.H. (2011). Talking with patients and families about medical error: a guide for education and practice. Baltimore: The Johns Hopkins University Press.

Weerakkody, R.A., Cheshire, N.J., Riga, C., Lear, R., Hamady, M.S., Moorth, K., Darz, A.W., Vincen, C., \& Bicknell, C.D. (2013). Surgical technology and operating-room safety failures: a systematic review of quantitative studies. BMJ Quality \& Safety, 22(9), 710-718.

Wu, A.W., McCay, L., Levinson, W., Iedema, R., Wallace, G., Boyle, D.J., \& McDonald, T.B. Bismark, M.M., Kraman, S.S., Forbes, E., Conway, J.B., \& Gallagher, T.H. (2017). Disclosing adverse events to patients: International norms and trends. Journal of Patient Safety, 13(1), 43-49. 